\title{
Langzeitverlauf der Juvenilen Idiopathischen Arthritis
}

Tollisen A et al. Longitudinal Health Status from Early Disease to Adulthood and Associated

Prognostic Factors in Juvenile Idiopathic Arthritis. J Rheumatol 2019; 46: 1335-1344. doi: 10.3899/ jrheum. 180948

Ein erheblicher Teil der Patienten mit einer Juvenilen Idiopathischen Arthritis (JIA) leidet bis ins Erwachsenenalter an Krankheitsmanifestationen. Angesichts der modernen Behandlungsoptionen stellt sich die Frage nach dem LangzeitKrankheitsverlauf und welche Parameter diesen vorhersagen. Norwegische Wissenschaftler versuchen diese Wissenslücke mithilfe einer Longitudinalstudie zu füllen.

Sie berichten über 96 Patienten, die zwischen 1995 und 1999 an der Universität Oslo aufgrund einer JIA behandelt worden waren. Zwischen der Diagnose und dem Studieneinschluss waren höchstens 18 Monate vergangen. Die Kinder (67\% Mädchen) waren im Schnitt 6,1 $\pm 4,0$ jahre alt und wurden in den folgenden 3 Jahren halbjährlich nachuntersucht. Im Rahmen dieser Termine erfassten die behandelnden Ärzte die Krankheitsaktivität, die funktionelle Leistungsfähigkeit sowie Schmerzen, Fatigue und das allgemeine Wohlbefinden der Patienten. Etwa 19 Jahre später stellten sich die Patienten zu einer erneuten Nachuntersuchung vor. Sie hatten inzwischen ein Alter von durchschnittlich 25,1 \pm 4 , 2 Jahren erreicht. Neben demografischen Informationen erfassten die Forscher hier mithilfe des „Medical Outcomes Study 12 -item Short Form Health Survey/Version 2“ ihre physische und psychische gesundheitsbezogene Lebensqualität sowie mithilfe des „Health Assessment Questionnaire-Disability Index (HAQ-DI)“ körperliche Einschränkungen. Schmerzen, Fatigue und das allgemeine Wohlbefinden waren erneut Teil der Erhebung. Das Vergleichskollektiv bildeten 96 bezüglich des Alters und des Geschlechts gematchte gesunde Personen aus der Allgemeinbevölkerung. 


\section{Ergebnisse}

Während der ersten 3 Nachbeobachtungsjahre nahmen die Krankheitsaktivität und der Grad der Einschränkungen signifikant ab und der Anteil der JIA-Patienten mit dem bestmöglichen Wohlbefinden nahm signifikant zu. Die Schmerzstärke und die Fatigue veränderten sich in diesem Zeitraum dagegen nicht wesentlich. Nach 19 Jahren beobachteten die Forscher ähnlich starke Schmerzbelastungen sowie Einschränkungen der körperlichen Leistungsfähigkeit und des Wohlbefindens wie nach 3 Jahren, der Grad der Erschöpfung hatte allerdings im Zeitverlauf signifikant zu- und der Anteil der Patienten ohne körperliche Defizite signifikant abgenommen. 19 Jahre nach Studieneinschluss wiesen die JIA-Patienten im Vergleich zu den Kontrollen eine deutlich stärkere Schmerzbelastung und Fatigue sowie eine signifikant schlechtere physische gesundheitsbezogene Lebensqualität auf. Bezüglich der psychischen Komponente der Lebensqualität unterschieden sich die Patienten und die Gesunden dagegen nicht wesentlich. Die multivariate Analyse ergab: Anhand der Anzahl betroffener Gelenke und der körperlichen Einschränkungen bei Studieneinschluss sowie der Einschränkungen nach 3 Jahren ließ sich die Schmerzintensität nach 19 Jahren vorhersagen. Bezüglich der physischen gesundheitsbezogenen Lebensqualität nach 19 Jahren erwiesen sich die Schmerzbelastung und die Anzahl betroffener Gelenke bei Studieneinschluss sowie die Anzahl betroffener Gelenke und die körperlichen Einschränkungen nach 3 Jahren als prädiktiv.

FAZIT

Die JIA geht über einen langen

Zeitraum mit einer stabilen körperlichen Leistungsfähigkeit, Wohlbefinden sowie Schmerzbelastungen einher, schlussfolgern die Autoren. Bis zum Erwachsenenalter nehmen allerdings Erschöpfungssymptome zu und die Patienten weisen im Vergleich zu Gesunden eine reduzierte Gesundheit auf. Schmerzen, die Gelenkaktivität sowie körperliche Einschränkungen scheinen dabei frühe Prädiktoren für einen ungünstigen Krankheitsverlauf darzustellen.

Dr. med. Judith Lorenz, Künzell 